\title{
Two-phase modelling for sediment water mixtures above the limit deposit velocity in horizontal pipelines
}

\author{
Thijs Schouten*, Cees van Rhee, Geert Keetels \\ Section Offshore and Dredging Engineering, Delft University of Technology, Mekelweg 2, Delft, Netherlands. \\ * Corresponding author. Tel.: +31 1527 84461. E-mail: T.D.Schouten@tudelft.nl
}

\begin{abstract}
In dredging applications, deep sea mining and land reclamation projects typically large amounts of sediments are transported through pipes in the form of hyper concentrated (40\% sediment or more) sediment-water mixtures or slurries. In this paper it is investigated how well a generic Euler-Euler CFD-model is capable to model velocity, concentration profiles and the pressure gradient of sediment above deposition limit velocity in a pipeline. This EulerEuler solver treats both phases as a continuum with its own momentum and continuity equations. The full kinetic theory for granular flows is accounted for (no algebraic form is used) and is combined with a buoyant $k-\varepsilon$ turbulence model for the fluid phase. The influence of the mesh size has been checked and grid convergence is achieved. All numerical schemes used are of second-order accuracy in space. The pressure gradient was calibrated by adjusting the specularity coefficient in one calibration case and kept constant afterwards. Simulations were carried out in a wide range of slurry flow parameters, in situ volume concentration (9-42\%), pipe diameter $(0.05-0.90 \mathrm{~m})$, particle diameter $(90-440 \mu \mathrm{m})$ and flow velocity of $(3-7 \mathrm{~m} / \mathrm{s})$. The model shows satisfactory agreement to experimental data from existing literature.
\end{abstract}

Keywords: Slurry flow; Two-phase modelling; CFD; Kinetic theory.

\section{INTRODUCTION}

In dredging applications, deep sea mining and land reclamation projects large amounts of sediments are transported through pipes. Hyper concentrated sand-water mixtures or slurries are pumped through the system where the volume concentration of the sediments can be as high as $40 \%$. In this paper we focus on flows where the bulk flow velocity is well above the limit deposit velocity. The limit deposit velocity is defined here as the bulk flow velocity where there is no stationary bed or sliding bed. The goal of this publication is to assess the performance of a generic Euler-Euler model to be able to predict the velocity, the concentration distribution and the pressure gradient correctly.

The transport of slurries has been researched since the third decade of the 20th century. Rouse (1937) and O'Brien (1933) predicted the concentration distribution of low concentrated slurries flowing through an open channel with a diffusion model. Some modifications to this model were made by Ismail (1952) who correlated the shear velocity gradient to the coefficient of mass transfer. Many other researchers proposed models to predict the concentration profile for slurry flows. These researchers are Shook and Daniel (1965), Shook et al. (1968), Gillies and Shook (1994), Gillies and Shook (2000) and Kaushal et al. (2003).

Apart from the concentration profiles, another aspect of flows through a pipeline is the horizontal pressure gradient. This is important as it relates to the energy loss and hence the required power supply. Several semi-empirical models exist that predict the pressure loss as a function of the bulk velocity and concentration for flow above the deposit limit (Durand (1953), Kaushal and Tomita (2003), Kaushal et al. (2005), Matousek (2002), Matousek (2009), Turian and Yuan (1977), Schaan et al. (2000), and Wilson et al. (2002)). Increase in computer power makes simulations with Computational Fluid Mechanics (CFD) software more attractive.
For the continuum modelling of a fluid with dispersed solid particles two main approaches exist. Firstly, the EulerLagrangian approach, where individual particles are tracked in a continuum of liquid. The movement of the particles is determined by the forces acting on the particle. Secondly, the Eulerian approach, in which both the fluid and the dispersed particles are treated as a continuum. This can be split in two methods of which the first is the Euler-Euler approach where the particle as well as the fluid phase are both treated as a separate continuum. The second is the mixture approach where the combination of fluid and particles is seen as one mixture with associated density and viscosity. The relative motion of the particles to the carrier fluid requires a closure relation and the sediment fraction requires an additional convection diffusion equation.

The Euler-Lagrangian approach offers a detailed model of the dispersed particles. Behaviour due to particle-particle interaction follows directly from the model. The computational costs of this approach strongly increase with the number of particles.

The mixture approach is the least expensive method. The simplification of a fluid with one mixture density and viscosity and an advection-diffusion equation can be combined with a rheological approach to include high concentration regions where particle-particle interaction becomes important (Goeree et al., 2016). The difficulty of this method is that the results depends on a fit on rheological measurements which are obtained in steady-state and homogeneous suspension for limited shear-rates (Boyer et al., 2011).

Several efforts have been made to simulate slurry flow with CFD by other authors. Ekambara et al. (2009) used a transient three-dimensional model that simulates slurries of sand with a two-phase model with the kinetic theory of granular flows for the sand fraction. Instead of solving for the full granular temperature equation a simplified algebraic expression was used for numerical stability reasons. A no-slip boundary condition was used for the particle phase at the pipe wall, which may not 
be valid. They investigated the effect of grain size on the concentration profile, solid and liquid velocity profiles and pressure loss. Comparison to experimental data with volume concentration ranging from $8 \%$ to $45 \%$, particle size $90 \mu \mathrm{m}$ up to $500 \mu \mathrm{m}$ and velocity 1.5 to $5.5 \mathrm{~m} / \mathrm{s}$ showed overall good agreement. Chen et al. (2009) modelled a coal-water slurry with an Eulerian multiphase approach based on the kinetic theory of granular flows. The coal-water slurry is modelled as a slurry with a bimodal distribution with two solid-phase fractions. Simulations were compared to experiments with coal-water slurries in pipelines with a diameter of $32 \mathrm{~mm}$ up to $50 \mathrm{~mm}$, concentrations from $30 \%$ to $53.8 \%$ and give fairly good results for the concentration distribution and the pressure. Kaushal et al. (2012) used a two-phase Euler-Euler approach and a mixture model to simulate a slurry of glass beads $(125 \mu \mathrm{m})$. Simulated results were compared the CFD results with experiments in a $54.9 \mathrm{~mm}$ diameter pipeline with velocities $1-5 \mathrm{~m} / \mathrm{s}$ and concentrations from 0 to $50 \%$. The pressure drop for clear water is in agreement for both models. However, if the concentration of sediment is increased the pressure drop calculated with the mixture model fails to predict this pressure gradient correctly. The Euler-Euler model gives good agreement for the pressure drop and good agreement for the concentration at the pipe centre. The predicted concentrations were overestimated at the bottom of the pipe and underestimated at the top pipe. Gopaliya and Kaushal (2015) simulated a three-dimensional sand-water slurry with a similar approach. Simulations were carried out for a $53 \mathrm{~mm}$ pipeline with velocities from $1.8-3.1 \mathrm{~m} / \mathrm{s}$ and volume concentrations of $15 \%-45 \%$ with grain size $0.18,0.28,0.55$ and $2.4 \mathrm{~mm}$. These simulations showed that at higher concentrations and increased grain sizes the solids concentration at the bottom of the pipe do not match the measurements. The highest solid concentration is close to the lower wall of the pipe but shifts upwards with increase of grain size. They observed secondary flow patterns in the vertical cross-sections. These result from the Reynolds-stresses that are not angular symmetric due to the presence of solid particles with a density that differs from the fluid phase. These secondary flow velocities increase when the grain size is increased. Gopaliya et al. (2016) performed a three-dimensional CFD analysis of two-phase slurry (sandwater) flows using the same approach as previous. Simulations were compared to experimental data of $263 \mathrm{~mm}$ pipelines with a velocity range of $3.5-4.7 \mathrm{~m} / \mathrm{s}$ and a concentration of $9.95 \%$ $34 \%$. The simulations showed reasonable agreement for concentration and velocity of the experimental results. The pressure gradient increases with increasing solid concentration and increasing velocity, but was not validated by experiments. Kumar et al. (2019) used a CFD code based on the Euler-Euler approach. The slurries that were simulated are iron-ore slurries with a mean diameter of $12 \mu \mathrm{m}$ through a $105 \mathrm{~mm}$ pipe with flow velocities ranging from $1.35-5.11 \mathrm{~m} / \mathrm{s}$. The simulations showed that the pressure drop deviates from experiments when a vertical concentration gradient develops. Ting et al. (2019) modelled the concentration distribution with two models, the empirical DHLLDV model and a two-phase Eulerian model similar to Kaushal et al. (2012). For the kinetic theory they used an algebraic expression. The turbulence in both phases is modelled with a mixture model. Simulations were compared to experimental data with in situ volume concentration $9 \%-42 \%$, pipe diameter 0.05 to $0.263 \mathrm{~m}$, particle diameter 90 to $440 \mu \mathrm{m}$ and flow velocity of 3 to $6.5 \mathrm{~m} / \mathrm{s}$. They found satisfactory agreement with measured concentration distributions. Messa and Matousek (2020) simulated slurry flow where all particles are kept in suspension by turbulence in horizontal pipes using the two-fluid model of Messa et al. (2014). The computational results were compared to experiments with pipe diameters $53.2-459 \mathrm{~mm}$, particles diameter ranging from 90 to $180 \mu \mathrm{m}$, velocities from 1.1 up to $6.0 \mathrm{~m} / \mathrm{s}$ and concentrations between $10 \%-36 \%$. They introduced an empirical parameter for the mixture viscosity which they include in their particle Reynolds number. They found satisfactory agreement with the experiments. They also demonstrated that in general, even in this simple geometry, many possible sources of inaccuracy and uncertainty come into play when simulating slurries. Presumably this holds for other Euler-Euler model approaches as well. In Messa et al. (2021) further insight was gained in this model by correlating the fluid dynamic solution with the mathematical structure of the two-fluid model. Results of slurry simulations between two horizontal plates were analysed in detail. The single terms in the streamwise momentum equations, namely, convection, within-phase diffusion, phase diffusion, pressureforce, and inter-phase friction force, were investigated. The impact of these simulations for circular pipe flow was explored afterwards acknowledging the difference with a 2D flow between horizontal flows.

The previous section shows that slurry flows through pipelines have been simulated with CFD. However, when the kinetic theory for granular flows was used an algebraic expression was used neglecting the advection and diffusion of granular temperature. Presumably these terms are important in the nearwall region with high gradients and shear and thus for the entire concentration distribution over the vertical cross-section. Another important term to include is the buoyancy destruction in the turbulence model for the fluid phase. This term includes the destruction of turbulent kinetic energy due to the concentration gradient. This term is not included in previous mentioned research. An existing generic formulated continuum model based on averaged equations for hyper concentrated sediment will be used for transport through a horizontal pipe line that solves for the granular temperature with advection and diffusion (no algebraic expression). The turbulence model includes the buoyancy destruction. Simulations will be compared to experimental data to show that the model is able to predict the pressure gradient, velocity and concentration profiles for cases with conditions with a bulk flow above the limit deposit velocity.

\section{Description of the model}

This paper deals with simulations of large diameter pipelines with a large amount of particles. The goal is to capture the macro effects in the pipeline and not the individual particles trajectories. For the reasons presented in the previous section the Euler-Lagrangian and the mixture approach do not meet these criteria and an Euler-Euler approach is chosen which models the particle-particle interaction but does not track every single particle and is therefore less expensive than an EulerLagrangian approach.

\section{Description of the Two Phase Model}

TwoPhaseEulerFoam is a solver that is available in the CFD package OpenFOAM (Greenshields, 2015). This is an open source package which allows for insight in the code and adjustment addition of code easily. In this paper the readily available solver with newly implemented functions for the drag and the fluid velocity has been used. The solver is based on the Euler-Euler approach and therefore assumes that the slurry flow consists of a fluid phase, $f$, and a solid phase, $s$, which form inter-penetrating continua. The volumetric concentration of the fluid phase is denoted as $\alpha_{f}$ and of the solid phase as $\alpha_{s}$ which 
together must be $\alpha_{f}+\alpha_{s}=1$. For each individual phase the laws of conservation of mass and momentum are satisfied. The coupling between the phases is accomplished by a continuous pressure field and coupling forces such as the drag force. The code of TwoPhaseEulerFoam (OpenFOAM 4.x) is based on the code of van Wachem (2000). The continuity and momentum equation for each phase are given by

$$
\frac{\partial \alpha_{k} \rho_{k}}{\partial t}+\nabla \cdot\left(\alpha_{k} \rho_{k} \mathbf{u}_{k}\right)=0
$$

and

$$
\frac{\partial \alpha_{k} \rho_{k} \mathbf{u}_{k}}{\partial t}+\nabla \cdot\left(\alpha_{k} \rho_{k} \mathbf{u}_{k} \mathbf{u}_{k}\right)=-\alpha_{k} \nabla P_{k}+\nabla \cdot \alpha_{k} \boldsymbol{\tau}_{k}+\alpha_{k} \rho_{k} \mathbf{g}+\mathbf{M}_{k}
$$

The subscript, $k$, indicates the phase, $P_{k}$ is the pressure in phase $k$. In solid-fluid mixtures this can be split into the pressure contributions due to the pressure of the continuous phase, $p_{f}$, and a pressure due to collision and enduring contact between the suspended particles, $p_{p}$. Wachem (2000)

$$
P_{k}=p_{f}+p_{p}
$$

The averaging process has introduced an extra term, $\boldsymbol{\tau}_{k}^{\mathrm{Re}}$, in the total shear stress component of the momentum equation, $\boldsymbol{\tau}_{k}=\boldsymbol{\tau}_{k}^{v i s}+\boldsymbol{\tau}_{k}^{\mathrm{Re}}$. The term $\boldsymbol{\tau}_{k}^{\mathrm{Re}}$ represents the effect of turbulent fluctuations on the main motion. The total stress term, $\tau_{k}$, the coupling forces between phases, $\mathbf{M}_{k}$, and the particle pressure, $p_{p}$, need closure and will be discussed in the following sections.

\section{Buoyant $k-\varepsilon$ model}

For the fluid phase a buoyant $k-\varepsilon$ model is used to model the turbulence (Rodi, 1980). This model is an adaptation of the standard $k-\varepsilon$ model of Launder and Spalding (1974). In this formulation the effect of density gradients is taken into consideration. The equation for the turbulent kinetic energy $k$ is

$\frac{\partial\left(\alpha_{f} \rho_{f} k_{f}\right)}{\partial t}+\nabla \cdot\left(\alpha_{f} \rho_{f} k_{f} \mathbf{u}_{f}\right)=\nabla \cdot\left(\mu+\frac{\mu_{t}}{\sigma_{k}}\right) \nabla k+P_{k}-P_{b}-\rho \epsilon_{f}$

The dissipation of turbulent kinetic energy is described with

$$
\begin{aligned}
& \frac{\partial\left(\alpha_{f} \rho_{f} \epsilon_{f}\right)}{\partial t}+\nabla \cdot\left(\alpha_{f} \rho_{f} \epsilon_{f} \mathbf{u}_{f}\right)= \\
& =\nabla \cdot\left(\mu+\frac{\mu_{t}}{\sigma_{\epsilon}}\right) \nabla \epsilon+C_{1} \frac{\epsilon}{k}\left(P_{k}+\left(1-C_{3}\right) P_{b}\right)-C_{2} \rho_{f} \frac{\epsilon_{f}^{2}}{k}
\end{aligned}
$$

where $\sigma_{k}$ and $\sigma_{\epsilon}$ are the turbulent Prandtl numbers, $C_{1}, C_{2}$ and $C_{3}$ are empirical coefficients. These are taken to be the same as reported by Henkes et al. (1991). The turbulent production of kinetic energy, $P_{k}$, is defined by the following equation

$$
P_{k}=2 \mu_{t} \mathbf{E}: \mathbf{E}
$$

where $\mathbf{E}$ is the strain rate tensor. The buoyancy destruction of turbulent kinetic energy, $P_{b}$ reads

$$
P_{b}=\frac{C_{\mu} k}{\epsilon} \mathbf{g} \cdot \nabla \rho
$$

where $C_{\mu}$ is a chosen constant. The values of $C_{\mu}, C_{1}, C_{2}, C_{3}$, $\sigma_{k}$ and $\sigma_{\varepsilon}$ are listed in Table 1 .

And the eddy viscosity $\mu_{t}$ is calculated as

$\mu_{t}=\rho C_{\mu} \frac{k^{2}}{\epsilon}$

Now the viscous shear stress and the turbulent shear stress can be combined into one equation for the total shear stress,

$$
\nabla \cdot\left(\boldsymbol{\tau}_{f}{ }^{v i s c}+\boldsymbol{\tau}_{f}^{R e}\right)=\nabla \cdot \mu_{\text {total }}\left[\left(\nabla \mathbf{u}+\nabla \mathbf{u}^{T}\right)-\frac{2}{3} \mathbf{I}(\nabla \cdot \mathbf{u})\right]
$$

where

$\mu_{\text {total }}=\mu_{f}+\mu_{t}$

Table 1. Coefficients for turbulence model.

\begin{tabular}{|c|c|c|c|c|c|}
\hline$C_{\mu}$ & $C_{1}$ & $C_{2}$ & $C_{3}$ & $\sigma_{k}$ & $\sigma_{\varepsilon}$ \\
\hline 0.09 & 1.44 & 1.92 & -0.33 & 1 & 1.3 \\
\hline
\end{tabular}

\section{Kinetic theory for dispersed particles}

For the solid phase stress, a different closure is used. Particles that are in suspension where their motion is dominated by collisional interaction the concepts from the kinetic theory for gasses can be used to describe the collisional stresses in the solid phase. The relations that describes the stresses due to kinetic energy transferred by particle velocity fluctuations and collisional behaviour of the solid phase have been derived by Lun (1991) based on the kinetic theory.

The total stress in the particle phase

$$
\nabla \boldsymbol{\tau}_{s}=\nabla \mu_{s}\left[\left(\nabla \mathbf{u}+\nabla \mathbf{u}^{T}\right)-\frac{2}{3} \mathbf{I}(\nabla \cdot \mathbf{u})\right]+\lambda_{s}(\nabla \cdot \mathbf{u I})
$$

where $\lambda_{s}$ is the bulk viscosity of the solid, $\mathbf{I}$ is the identity tensor. If compared to the shear stress for the fluid phase 1.10 it can be seen that this shear stress has an extra component. This extra component is the bulk viscosity of the solid phase and represents the added viscosity due to the resistance of compression of the solid phase. This bulk viscosity of the solids is given by

$$
\lambda_{s}=\frac{4}{3} \alpha_{s} \rho_{s} d_{p} g_{0}\left(1+e_{s s}\right) \sqrt{\frac{\Theta_{s}}{\pi}}
$$

where $d_{p}$ is the particle diameter, $g_{0}$ is the radial distribution function, $e_{s s}$ is the restitution coefficient and $\Theta_{s}$ is the granular temperature. The radial distribution function, $g_{0}$, is employed to describe the probability of finding a particle at a certain distance from a reference particle. This is a correlation factor that modifies the probability of collisions between grains when the solid phase becomes denser and can be regarded as a measure for the probability of inter-particle contact

$g_{o}=\left[1-\left(\frac{\alpha_{s}}{\alpha_{s, \max }}\right)\right]^{-1}$

This is the equation as proposed by Bagnold (1954) where 
$\alpha_{s, \max }$ is the maximal concentration at random packing density.

The solids shear viscosity, $\mu_{s}$, accounts for the tangential forces on the particles. Multiple variants of this equation exist, in this work the equation that is used is as proposed by Gidaspow (1994). This equation does not account for the inelastic nature of the particles claiming this correction is negligible, the validity of this claim is shown in Wachem (2000). The formulation of Syamlal et al. (1993) neglects the kinetic contribution which is dominant in dilute regions. The formulation of Gidaspow (1994) includes this contribution and therefore is used

$\mu_{s}=\frac{4}{5} \alpha_{s}^{2} d_{p} g_{0}\left(1+e_{s s}\right) \sqrt{\frac{\Theta}{\pi}}+\frac{2 \frac{5 \sqrt{\pi}}{96} \rho_{s} d_{p} \sqrt{\Theta}}{\left(1+e_{s s}\right) g_{0}}\left[1+\frac{4}{5} g_{0} \alpha_{s}\left(1+e_{s s}\right)\right]^{2}$

The granular temperature is introduced as a measure for the particle velocity fluctuations

$\Theta_{s}=\frac{1}{3} \overline{v_{s}^{\prime 2}}$

The solid phase stress depends on the magnitude of these velocity fluctuations. Therefore, a balance is required that is associated with these particle velocity fluctuations. This balance is given as

$$
\begin{aligned}
& \frac{3}{2}\left[\frac{\partial}{\partial t}\left(\alpha_{s} \rho_{s} \Theta_{s}\right)+\nabla \cdot\left(\alpha_{s} \rho_{s} \Theta_{s} \mathbf{u}_{s}\right)\right]= \\
& =\left(-P_{s} \mathbf{I}+\boldsymbol{\tau}_{s}\right): \nabla \mathbf{u}_{s}-\nabla \cdot\left(\kappa_{s} \nabla \Theta_{s}\right)-\gamma_{s}-J_{s}
\end{aligned}
$$

The first term on the right hand side represents the creation of fluctuation energy due to the shear in the solid phase. The second term represents the dissipation of fluctuation energy along gradients of the granular temperature. $\gamma_{s}$, represents the dissipation of granular temperature due to the inelastic collisions between particles and $J_{s}$ represents the dissipation or creation of granular energy due to the transfer of kinetic energy of random fluctuations in particle velocity.

The pressure that is the result of the solid-phase normal forces due to particle-particle collisions is called the particle pressure, $P_{s}$. There is general agreement in literature on the form of the particle pressure and is given by Lun (1991) as

$$
P_{s}=\alpha_{t} \rho_{t} \Theta_{s}\left(1+2 g_{0} \alpha_{t}\left(1+e_{s s}\right)\right)
$$

In Equation (1.16) $\kappa_{s}$ is the diffusion coefficient corresponding to the dissipation of fluctuation energy and consist of a kinetic contribution and a collisional contribution (Gidaspow, 1994)

$$
\kappa_{s}=\frac{1}{\left(1-e_{s s}\right) g_{0}}\left[1+\frac{6}{5}\left(1+e_{s s}\right) g_{0} \alpha_{s}\right]^{2} \kappa_{d i l}+2 \alpha_{s}^{2} \rho_{s} d_{p} g_{0}\left(1+e_{s s}\right) \sqrt{\frac{\Theta_{s}}{\pi}}
$$

where $\kappa_{d i l}$ is the diffusion coefficient in the limit of dilute suspensions

$$
\kappa_{d i l}=\frac{75}{385} \rho_{s} d_{p} \sqrt{\pi \Theta_{s}}
$$

The dissipation of granular energy, $\gamma_{s}$, due to particleparticle collisions is described by Jenkins and Savage (1983). This represents the rate of energy dissipation within the particle phase due to collisions between the particles. In Lun (1991) the term $\nabla \cdot u_{s}$ is omitted which is typically permitted when the mean-field gradients associated with a slight particle inelasticity are small, this results into the following expression:

$$
\gamma_{s}=12(1-e)^{2} g_{0} \alpha_{s}^{2} \rho_{s} \frac{1}{d_{p}} \sqrt{\frac{\Theta_{s}}{\pi}}
$$

the last term, $J_{s}$, represents the transfer of the kinetic energy of random fluctuation in particle velocity from the particle phase to the fluid phase and is represented as proposed by Louge et al. (1991) based on the work of Koch (1990)

$$
J_{s}=3 K_{d}+\frac{K_{d} d_{p}\left(\mathbf{u}_{f}-\mathbf{u}_{s}\right)^{2}}{4 \alpha_{s} \rho_{s} \sqrt{\pi \Theta_{s}}}
$$

where $K_{d}$ is the inter-phase drag constant.

\section{Frictional stresses}

At solid fractions that are higher than a specific threshold $\alpha_{s, \text { min }}$ sustained contact between the particles starts to play a role. The particle collisions are no longer instantaneous at these concentrations and the frictional stresses that occur need to be accounted for in the solid phase stress (Zhang and Rauenzahn,1997). The frictional stresses are added to the stresses that are predicted by the kinetic theory. Multiple approaches have been presented in literature. The fictional stresses are typically expressed in a Newtonian form

$$
\boldsymbol{\sigma}_{s}=P_{s} \mathbf{I}+\mu_{s}\left(\nabla \mathbf{v}_{s}+\left(\nabla \mathbf{v}_{s}\right)^{T}\right)
$$

If the concentration $\alpha_{s}>\alpha_{s, \max }$ the frictional stress will be added to the stress predicted by the kinetic theory.

$$
\begin{aligned}
& P_{p}=P_{\text {kinetic }}+P_{\text {frictional }} \\
& \mu_{s}=\mu_{\text {kinetic }}+\mu_{\text {frictional }}
\end{aligned}
$$

A semi-empirical model for the normal frictional stress, $P_{f}$ was proposed by Johnson and Jackson (1987)

$$
P_{\text {frictional }}=\operatorname{Fr} \frac{\left(\alpha_{s}-\alpha_{s, \min }\right)^{n}}{\left(\alpha_{s, \max }-\alpha_{s}\right)^{m}}
$$

where $\alpha_{s, \text { min }}$ is the fraction at which contacts are no longer instantaneous but exert some frictional behaviour and $\alpha_{s, \max }$ is the packing density and $F r, n$ and $m$ are empirical constants depended on the material. The shear viscosity is related to the frictional normal stress by the well-known law as proposed by Coulomb

$\mu_{f}=P_{f} \sin (\phi)$

where $\phi$ is the angle of internal friction. 


\section{Interphase forces}

The last term of Equation (1.2), $\mathbf{M}_{k}$, represents the momentum transfer from the fluid phase to the particle phase and viceversa. The momentum transfer between the phases can be separated into four different forces and read

$\mathbf{M}_{k}=\mathbf{M}_{D}+\mathbf{M}_{L}+\mathbf{M}_{T D}+\mathbf{M}_{\text {lub }}$

where $\mathbf{M}_{D}$ is the drag force, $\mathbf{M}_{L}$ is the lift force, $\mathbf{M}_{T D}$ is the turbulent dispersion force and $\mathbf{M}_{\text {lub }}$ is the lubrication force.

\section{Drag force}

The drag force is modelled with the following procedure. The generalized force per unit of volume of suspension of particles can be written as

$\mathbf{M}_{D}=n_{p} \mathbf{F}_{d}$

where $\mathbf{M}_{D}$ is the force on the suspension of particles, $\mathbf{F}_{d r}$ is the drag force on a single particle

$\mathbf{F}_{d r}=\frac{1}{2} C_{d} A \rho \mathbf{u}^{2}{ }_{r}$

and $n_{p}$ is the number of particles per unit volume

$n_{p}=6\left(1-\alpha_{f}\right) / \pi d_{p}^{3}$

If we then take Equation (1.30) which represents the drag force acting on a single particle in the suspension and multiply it with equation (1.31) the drag force contribution per unit volume can be written as:

$n_{p} \mathbf{F}_{d r}=\frac{3}{4 d_{p}}\left(1-\alpha_{f}\right) C_{d} \rho_{f}\left|\mathbf{u}_{r}\right| \mathbf{u}_{r}$

This drag force can be written in a more generalized form as:

$n_{p} \mathbf{F}_{d r}=K_{d} \mathbf{u}_{r}$

where $K_{d}$ is the drag function. In Equation (1.31) $K_{d}$ is the drag function related to the unhindered settling velocity of a particle. In reality the settling velocity of the particles decrease when the concentration of particles increase. To adjusts for hindered settling effects the drag function is modelled according to the model of Di Felice (1994)

$$
K_{d}=\frac{3}{4 d_{p}}\left(1-\alpha_{f}\right) C_{d} \rho_{f}\left|\mathbf{u}_{r}\right| \alpha_{f}^{2-\beta}
$$

where $C_{d}$ is the drag coefficient given by DallaValle (1948)

$C_{d}=\left(0.63+4.8 \sqrt{\frac{1}{R e_{p}}}\right)^{2}$

Di Felice showed that the exponent $\beta$ depends on the particle Reynolds number

$\beta=3.7-0.65 e^{-\left(1.5-\log _{10}\left(R e_{p}\right)\right)^{2} / 2}$ where the particle Reynolds number is the Reynolds number of the instantaneous relative velocity

$R e_{p}=\frac{\left(\left|\mathbf{u}_{f}-\mathbf{u}_{s}\right|\right) \rho_{f} d_{p}}{\mu_{f}}$

This differs from the approach of Messa and Matousek (2020) who use the mixture viscosity in their formulation of the particle Reynolds number.

\section{Lift force}

A particle that moves in a shear flow and more generally in a rotating flow will experience a force transverse to its movement. This force is usually called the lift force. The lift force for small rigid particles was first observed by Segre and Silberberg (1962). The lift force can generally be described by the formulation of Auton (1987)

$\mathbf{F}_{l p}=C_{l} \rho_{f} V_{p}\left(\mathbf{u}_{r} \times \boldsymbol{\omega}_{f}\right)$

where $C_{l}$ is the lift coefficient, $V_{p}$ is the volume of a single particle, $\mathbf{u}_{r}$ is the relative velocity $\mathbf{u}_{f}-\mathbf{u}_{s}$ and $\boldsymbol{\omega}_{f}$ is the vorticity of the continuous phase. Equation (1.37) represents the lift force acting on a single spherical particle. The generalized lift force per unit volume is the lift force per particle times the amount of particles presented per unit volume. With $n_{p} V_{p}=\alpha_{s}$ this can be written as

$\mathbf{F}_{l}=\alpha_{s} C_{l} \rho_{f}\left(\mathbf{u}_{r} \times \boldsymbol{\omega}_{f}\right)$

The lift coefficient is calculated with empirical relations derived by Legendre and Magnaudet (1998) and depends on the local shear rate of the fluid and the particle Reynolds number. Legendre and Magnaudet(1998) splits the lift coefficient in a high $\left(R e_{p}>5\right)$ and a low Reynolds $\left(R e_{p}<5\right)$ contribution and showed that for particle Reynolds numbers $>5$ the value of the lift coefficient is independent of the shear rate. This indicates that the magnitude of the lift force is directly proportional to the vorticity of the shear of the undisturbed flow. The empirical relation they propose for the high Reynolds contribution to the lift coefficient is therefore a function of the particle Reynolds number only

$C_{L}^{H i g h R e}\left(R e_{p}\right)=\frac{1}{2} \frac{1+16 R e_{p}^{-1}}{1+29 R e_{p}^{-1}}$

The low Reynolds component, contrary to the high Reynolds component, dependents on both the shear rate, $S r$, of the fluid and the particle Reynolds number. The lift coefficient for the low Reynolds component can be written as

$C_{L}^{L o w R e}\left(R e_{p}, S r\right)=\frac{6}{\pi^{2}}\left(R e_{p}, S r\right)^{-\frac{1}{2}} J^{\prime}(\varepsilon)$

The function $J^{\prime}(\varepsilon)$ is an empirical adjusted version of a three-dimensional integral, $J(\varepsilon)$, by McLaughlin (1991). The function is determined in such a way that it agrees with results obtained from DNS simulations and has the following form

$J^{\prime}(\varepsilon)=\frac{J(\infty)}{\left(1+0.2 \varepsilon^{-2}\right)^{3 / 2}}$ 
where $J(\infty)=2.255$, and $\varepsilon$ is an function of the particle Reynolds number and the shear rate $(\mathrm{Sr})$

$\varepsilon=\sqrt{S r / R e_{p}}$

Finally, Equations (1.40) and (1.41) can be combined in an empirical expression that is valid for every particle Reynolds number. The simple but accurate expression is given by

$C_{L}\left(R e_{p}, S r\right)=\sqrt{\left[C_{L}^{L o w R e}\left(R e_{p}, S r\right)\right]^{2}+\left[C_{L}^{H i g h R e}\left(R e_{p}\right)\right]^{2}}$

Equation (1.44) is derived for a bubble dispersed inside a carrier fluid. Legendre and Magnaudet (1997) showed that the physical difference between a bubble and a solid sphere modifies the lift force with a numerical factor of $9 / 4$. Therefore, replacing the numerical factor of 6 in Equation (1.40) by 27/2 is sufficient for calculating the lift on solid particles.

\section{Dispersion force}

When a turbulent fluid flow interacts with the dispersed phase particles will tend to get caught by the turbulent eddies of the continuous phase. This results in a net particle transport from high to low concentration regions.

The turbulent dispersion force may be modelled using the time average of the fluctuating part of the inter phase momentum force. This idea was first proposed by Gosman et al. (1992) using only the drag component of the interfacial force. The influence of the lift force on the dispersion force has also been studied by Behzadi et al. (2004) but were found to be not significant. For the case of two-phase flow, the equation for the turbulent dispersion force can be described as follows, Burns et al. (2004).

$\mathbf{F}_{d}=K_{d} \frac{v_{t}}{\sigma_{a}}\left(\frac{1}{\alpha_{f} \alpha_{s}}\right) \nabla \alpha_{f}$

where $\sigma_{a}$ is the turbulent Prandtl number.

\section{Lubrication force}

When a spherical object moves towards a wall the water between the particle and the wall needs to flow away. The no-slip condition at the wall reduces the drainage of water between the particle and the wall which results in a force moving the particle away from the wall. To include this effect, the formulation proposed by Antal et al. (1991) is used

$\mathbf{F}_{l u b}=\frac{\alpha_{s} \rho_{f}\left|\mathbf{u}_{i}\right|^{2}}{0.5 d_{p}}\left[C_{F 1}+C_{F 2}\left(\frac{0.5 d_{p}}{y_{0}}\right)\right] \mathbf{n}_{w}$

where $C_{F 1}=-0.104-0.06 \mathbf{u}_{r}, C_{F 2}=0.147$, $\mathbf{u}_{i}=\left(\mathbf{u}_{s}-\mathbf{u}_{f}\right)-\left[\mathbf{n}_{w} \cdot\left(\mathbf{u}_{s}-\mathbf{u}_{f}\right)\right] \mathbf{n}_{w}$ and $\mathbf{n}_{w}$ is the wall normal vector.

\section{NUMERICAL SIMULATIONS Boundary conditions}

The inflow as well as the outflow of the domain have periodic boundary conditions. At the wall the following wall conditions have been used. In the fluid phase the wall is modelled according to the law of the wall. The particle phase is allowed to slip along the wall. Therefore, a partial slip condition is implemented. The amount of slip along the wall depends on the amount of particles, pressure on the particles and the specularity coefficient, $\varphi$, whose value depends on the roughness of the surface and varies between zero, for perfectly specular collisions, and unity, for perfectly diffuse collisions. To include this the model of Johnson and Jackson (1987) is used

$\mathbf{u}_{\text {wall }}=(1-c) \mathbf{u}_{s, 1}$

$c=\frac{\pi \alpha_{p} \rho_{s} g_{0} \phi \sqrt{3 \Theta_{s}}}{6 \alpha_{s, \max }}$

where $\mathbf{u}_{s, 1}$ is the velocity in the cell centre of the wall cell and $\mathbf{u}_{\text {wall }}$ is the velocity at the wall.

Since there is no theoretical approach to determine the specularity coefficient it needs to be chosen in a careful manner. The following procedure has been followed: a simulation was run and the specularity coefficient was adjusted until the pressure gradient was in agreement with experimental result. This procedure determined the specularity coefficient to be 0.1 . This value was then used in all following simulations. The reasoning for keeping the coefficient constant is that the roughness of the pipes, all metal tubing, is approximately the same. This coefficient together with other coefficients is listed in Table 2.

Table 2. Coefficients needed for simulation.

\begin{tabular}{|c|c|c|c|c|c|}
\hline$e_{s s}$ & $\phi$ & $\sigma_{a}$ & $\mathrm{Fr}$ & $\mathrm{m}$ & $\mathrm{n}$ \\
\hline 0.9 & 0.1 & 1 & 0.05 & 2 & 5 \\
\hline
\end{tabular}

\section{COMPUTATIONAL DOMAIN}

An example of the computational mesh for the simulations is shown in Figure 1. The size of the mesh changes per simulated case due to difference in pipe diameter, $D$. To exclude the effect of the mesh size on the overall solution simulations have been run on a coarse, medium and fine mesh. From this convergence study it was concluded that variation in the mesh size does not influence the predicted velocities, concentration profiles and pressure gradients. It was decided to run further simulations on the medium mesh for each case. The dimension of the cells at the wall is variable to account for the change in $\mathrm{y}^{+}$ due to change in fluid flow velocity. The $\mathrm{y}+$ is always maintained at a value of $\sim 100$ to remain within the limits, 80 to 200 , of the used log-law wall functions.

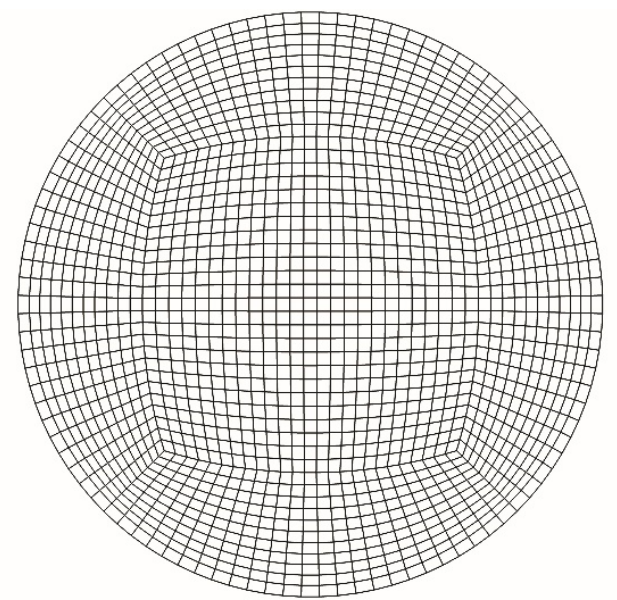

Fig. 1. Computational mesh for pipe simulations. 
Two-phase modelling for sediment water mixtures above the limit deposit velocity in horizontal pipelines

In the direction of the flow the domain has a periodic condition and has a domain length of 1 cell. The fluid phase in the domain is forced by a pressure function which applies a pressure difference to the fluid phase to maintain a prescribed average velocity.

\section{RESULTS AND ANALYSIS}

To assess the performance of the model that was presented in the previous section, simulations are compared to experimental results from literature. The cases were selected to have a sufficient variety of sediment sizes, pipe diameters and flow velocities above limit deposit velocity. Table 3 shows an overview of all experimental parameters. The first set of simulations represent the experimental work of Roco and Shook (1983) with dense sediment suspensions but still above the limit deposit velocity in $51.5 \mathrm{~mm}$ and $267 \mathrm{~mm}$ pipelines. The second set simulates the experiments by Gillies et al. (2004) which were perfomerd in a $103 \mathrm{~mm}$ pipeline with particle diameter $90 \mu \mathrm{m}$ and $270 \mu \mathrm{m}$. The third set simulates the experiments of Matousek (2002). The fourth set is compared with the results of Kaushal (2007) which consisted of experiments with $440 \mu \mathrm{m}$ particles in a $54.9 \mathrm{~mm}$ pipeline. The forementioned set of experiments is the same as were simulated in Ting et al. (2019) for the sake of comparison with Euler-Euler approach with algebraic approximation for the granular temperature. The last set of experiments is used to compare pressure gradients obtained by Silin et al. (1958) in a $0.9 \mathrm{~m}$ diameter pipeline which is a typical industrial sized pipeline.

In Figures 2-7 results for the concentration distribution as calculated by the model are compared to the corresponding measurements. When the concentration is measured with a radioactive source the computed concentration is the average along a horizontal line. In general, the agreement between experiments and computations is good in the middle section of the pipeline.

In Figures 2-5 the agreement between the measured concentration and the simulated concentration is good over the full height of the pipeline. A small deviation in particle concentration can be found in the utmost top and bottom wall of the pipeline. At these locations the simulated particle concentrations are lower than the measured concentrations. This might be attributed to the sensitivity of the simulation for wall related parameters such as the wall roughness and specularity coefficient. Also the limited accuracy of measurements with a radioactive source in the bottom and top $10 \%$ of the pipeline could have influenced the measured concentrations. The accuracy in the bottom and top region of the pipe is limited as the relative radiation absorption by the pipe wall itself is relatively high in these regions.

In Figure 6 the agreement between the simulated concentration and the measured concentration is not as good as the agreement found in the previous figures. Concentration in the bottom of the pipeline remains within a $10 \%$ deviation from the measured concentrations, but in the top of the pipe the simulat-ed concentration gives lower values than the measured concentration.
Figure 7 shows the simulated concentration for the experiments of Kaushal (2007). The simulated concentration does not show the same level of agreement as for the cases of Roco and Shook (1983) and Gillies et al. (2004). In all cases the simulated concentration in the bottom of the pipeline is higher. The higher concentration simulations show a relatively good agreement in the top of the pipeline but start to deviate in the bottom half of the pipe.

\section{Velocity distribution}

In Figure 8 the velocity distribution for the $3 \mathrm{~m} / \mathrm{s}, 19 \%$ concentration case from Gillies et al. (2004) is shown. Here the agreement between the simulations and experiments is within a $10 \%$ accuracy range. Unfortunately, velocity profiles for the other experiments mentioned in Table 3 are absent. More experiment data is needed to draw definitive conclusions about the accuracy of the computed velocity profiles. In qualitative comparison it was found that the maximum velocity was shifted from the pipe centre to a higher position. As the velocity is linked to the concentration profile is can be assumed that the velocity is correctly predicted as well.

\section{Hydraulic gradient}

Having a correct prediction of the pressure gradient in the simulations increases the confidence and practical value of the proposed approach. Figure 9 compares the measured pressure gradient with the computed value. It can be seen that the pressure gradient as predicted by the model remains within a $10 \%$ limit compared with the experiment. Figure 10 and 11 show the influence of the flow velocity and the concentration on the predicted hydraulic gradient and compares them to measured hydraulic gradients. It is seen that the trend of increasing hydraulic gradient with increasing flow velocity and concentration is correctly captures by the model.

Messa et al. (2014) found that the value of $y+$ can influence the predicted hydraulic gradient. For $y+<30$ they found the pressure gradient to be increasing and for $\mathrm{y}+>30$ to remain stable. To investigate this sensitivity in the present model simulations have been run with different cell sizes for the wall cell. Figure 12 shows the results from these additional simulations. It can be seen that within the limit of $80<y+<200$ the predicted gradient remains constant. As soon as the $y+$ is either lower or higher the predicted gradient is deviating.

As mentioned in Table 3, also simulations for greater, industrial sized pipelines were performed. In these cases, only the pressure gradient could be compared to experimental data since more detailed information was not available. The result of these simulations are compared to the experimental data in Figure 13. It can be seen that in this case the pressure gradient predicted by the model is still in the correct order of magnitude. This gives confidence that the model is capable of predicting the pressure drops correctly for 0.05 meter pipelines up to 0.900 meter pipelines.

Table 3. Experimental flow parameters used for computations.

\begin{tabular}{|c|c|c|c|c|c|}
\hline Experiment & $\mathrm{D}(\mathrm{m})$ & $\mathrm{D}_{\mathrm{p}}(\mathrm{mm})$ & $\mathrm{V}(\mathrm{m} / \mathrm{s})$ & $\alpha_{p}(\%)$ & $\rho_{\mathrm{p}}(\mathrm{kg} / \mathrm{m} 3)$ \\
\hline Gillies et al. (2004) & 0.103 & 0.09 & $2.00-7.00$ & $19-33$ & 2650 \\
\hline Gillies et al. (2004) & 0.103 & 0.270 & $1.00-7.00$ & $10-40$ & 2650 \\
\hline Roco and Shook (1983) & 0.0515 & 0.165 & $3.78-4.33$ & $9-29$ & 2650 \\
\hline Roco and Shook (1983) & 0.263 & 0.165 & 3.50 & $9.95-33.8$ & 2650 \\
\hline Matousek (2002) & 0.150 & 0.370 & 6.00 & $26-33$ & 2650 \\
\hline Kaushal (2007) & 0.0549 & 0.440 & 3.00 & $9.39-41.59$ & 2470 \\
\hline Silin et al. (1958) & 0.900 & 0.250 & $5.5-6.5$ & $11-20$ & 2585 \\
\hline
\end{tabular}




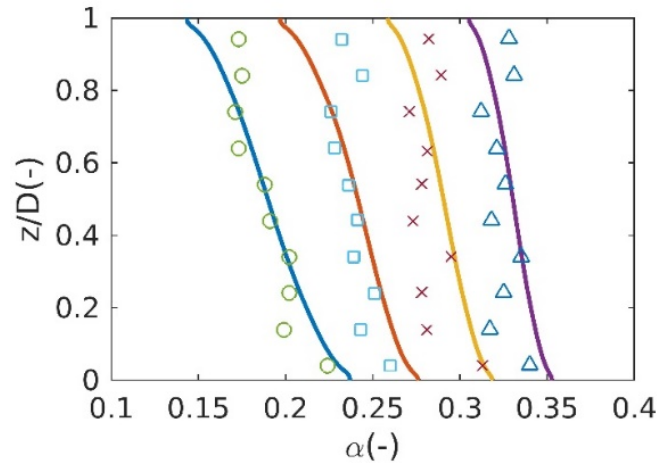

Fig. 2. Path averaged concentration profiles for Gillies et al (2004). $\mathrm{D}=0.103 \mathrm{~m}, \mathrm{dp}=0.090 \mathrm{~mm}$ and $\mathrm{V}=3 \mathrm{~m} / \mathrm{s}$. ० : $\alpha=19 \%$, 口: $\alpha=24 \%, \mathrm{x}: \alpha=29 \%, \Delta: \alpha=33 \%$, - simulations.

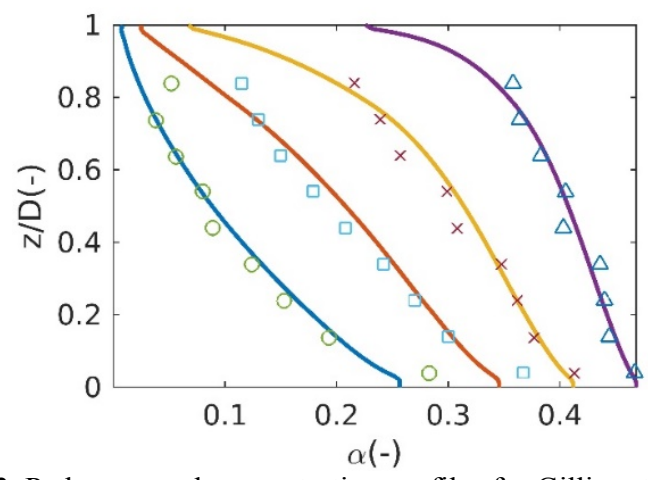

Fig. 3. Path averaged concentration profiles for Gillies et al (2004). $\mathrm{D}=0.103 \mathrm{~m}, \mathrm{dp}=0.270 \mathrm{~mm}$ and $\mathrm{V}=5.4 \mathrm{~m} / \mathrm{s} . \circ: \alpha=10 \%, \square: \alpha=$ $20 \%, \mathrm{x}: \alpha=30 \%, \Delta: \alpha=40 \%,-$ simulations.

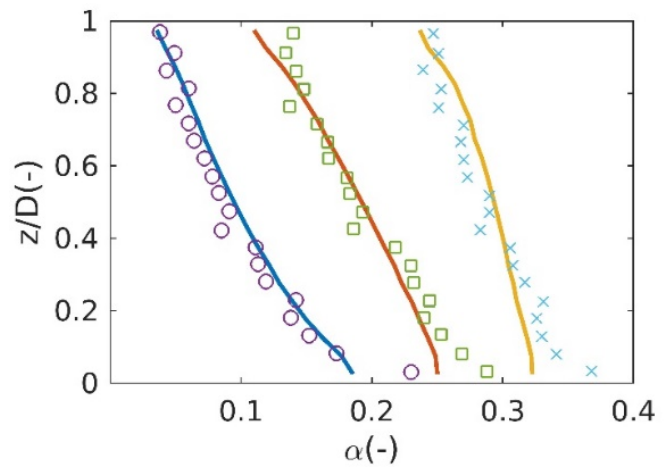

Fig. 4. Path averaged concentration profiles for Roco \& Shook (1990). $\mathrm{D}=0.0515 \mathrm{~m}, \mathrm{dp}=0.165 \mathrm{~mm}$ and $\mathrm{V}=3.79-4.33 \mathrm{~m} / \mathrm{s} . \circ: \alpha$ $=9 \%, \square: \alpha=19 \%, \mathrm{x}: \alpha=29 \%,-$ simulations.

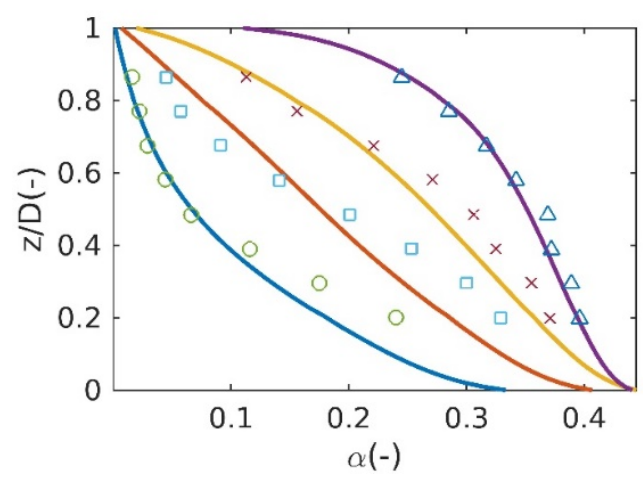

Fig. 5. Path averaged concentration profiles for Roco \& Shook (2004). $\mathrm{D}=0.263 \mathrm{~m}, \mathrm{dp}=0.165 \mathrm{~mm}$ and $\mathrm{V}=3.5 \mathrm{~m} / \mathrm{s} . \circ: \alpha=10 \%$, : $\alpha=18 \%, \mathrm{x}: \alpha=27 \%, \Delta: \alpha=34 \%,-$ simulations.

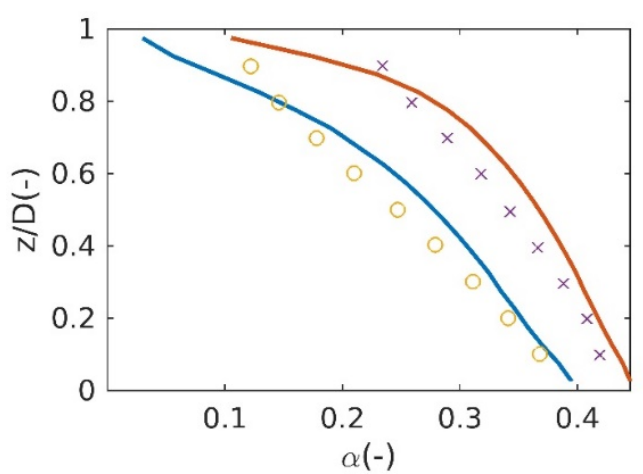

Fig. 6. Path averaged concentration profiles for Matousek (2002). $\mathrm{D}=0.150 \mathrm{~m}, \mathrm{dp}=0.370 \mathrm{~mm}$ and $\mathrm{V}=3 \mathrm{~m} / \mathrm{s} . \circ: \alpha=26 \%, \mathrm{x}: \alpha=$ $35 \%,-$ simulations.

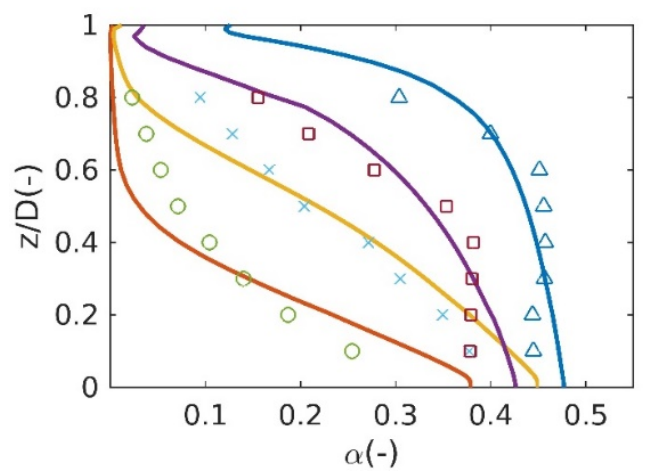

Fig. 7. Path averaged concentration profiles for Kaushal (2005). D $=0.0549 \mathrm{~m}, \mathrm{dp}=0.440 \mathrm{~mm}$ and $\mathrm{V}=3 \mathrm{~m} / \mathrm{s} . \circ: \alpha=9.39 \%$, $\square$ : $\alpha=21.68 \%$, x: $\alpha=30.1 \%, \Delta: \alpha=41.59 \%$, - simulations.

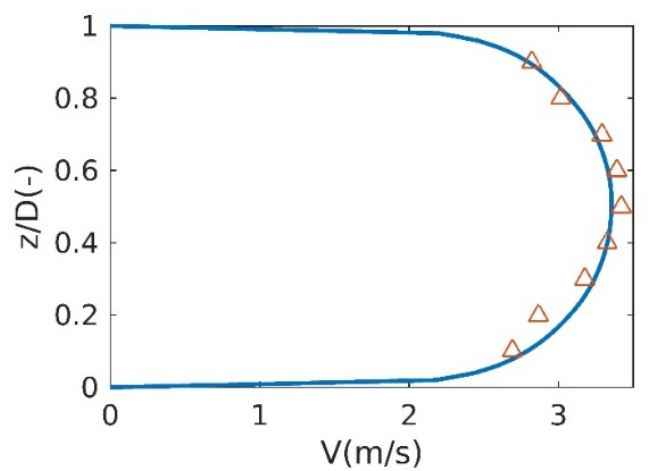

Fig. 8. Velocity profiles for Gillies et al. (2004): $\mathrm{D}=0.103 \mathrm{~m}, \mathrm{dp}=$ $0.090 \mathrm{~mm}$ and $\mathrm{V}=3 \mathrm{~m} / \mathrm{s}, \mathrm{Cv}=19 \%$. $\Delta$ : experiment, - : simulations.

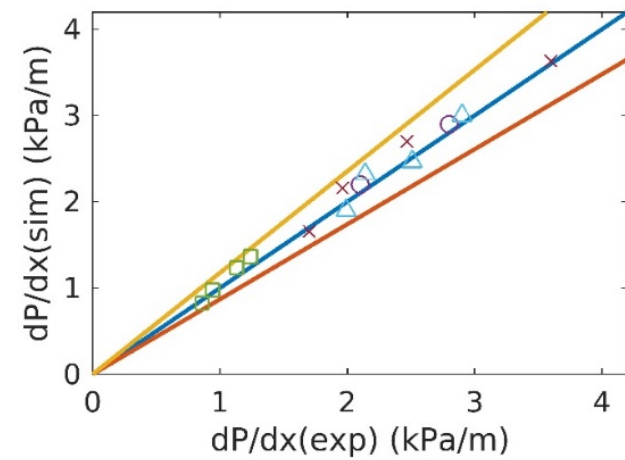

Fig. 9. Pressure drop comparison between experiment and simulations. $\square$ : Gillies et al. (2004) d $=0.090 \mathrm{~mm}, \Delta$ : Gillies et al. (2004) $\mathrm{d}=0.270 \mathrm{~mm}, \mathrm{x}$ : Kaushal (2005) $\mathrm{d}=0.440 \mathrm{~mm}$, ○: Matousek (2002) $\mathrm{d}=0.370 \mathrm{~mm}$. 


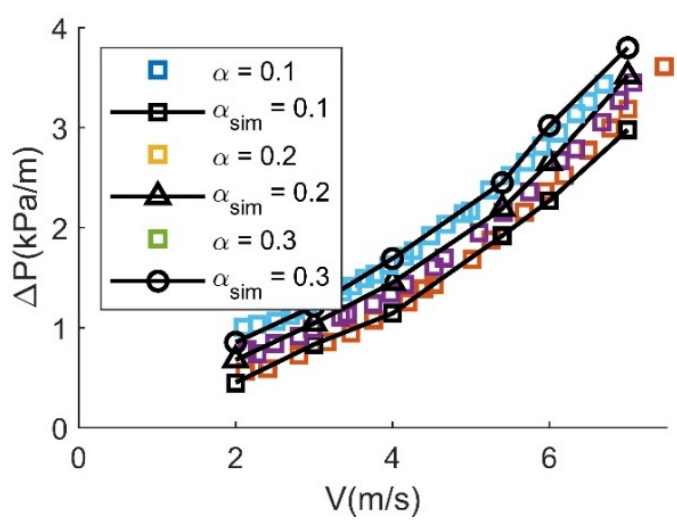

Fig. 10. Influence of concentration and flow velocity on hydraulic gradient for $90 \mu \mathrm{m}$ particles.

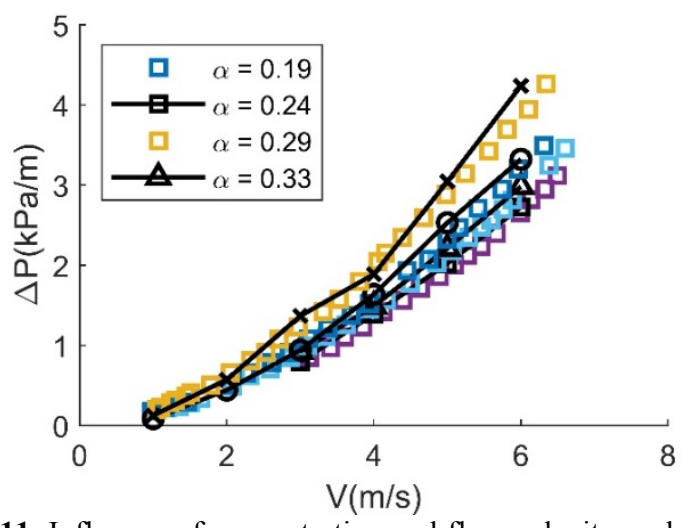

Fig. 11. Influence of concentration and flow velocity on hydraulic gradient for $270 \mu \mathrm{m}$ particles.

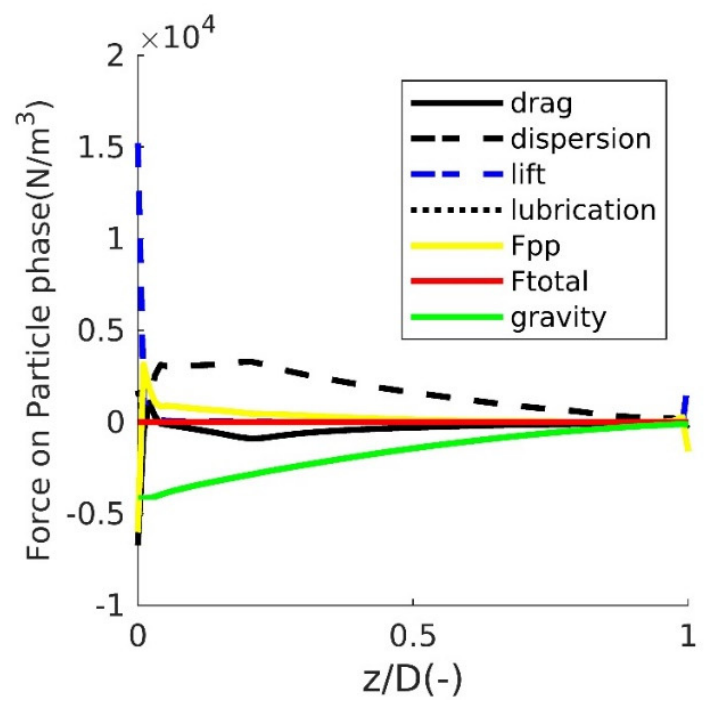

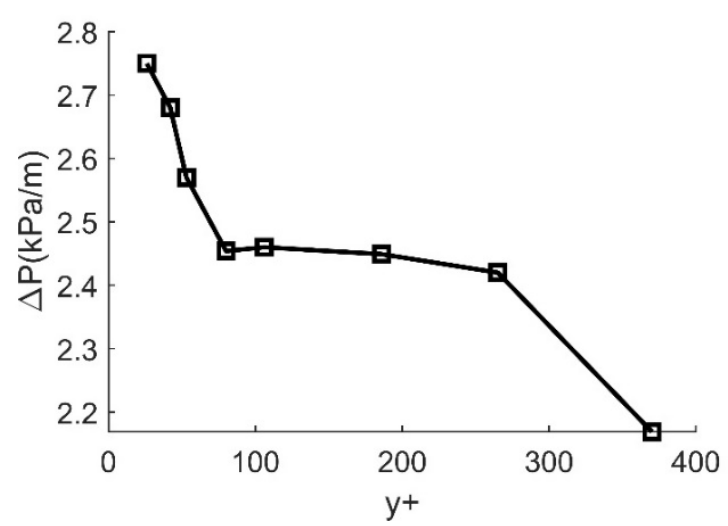

Fig. 12. Influence of $\mathrm{Y}+$ on the predicted pressure gradient.

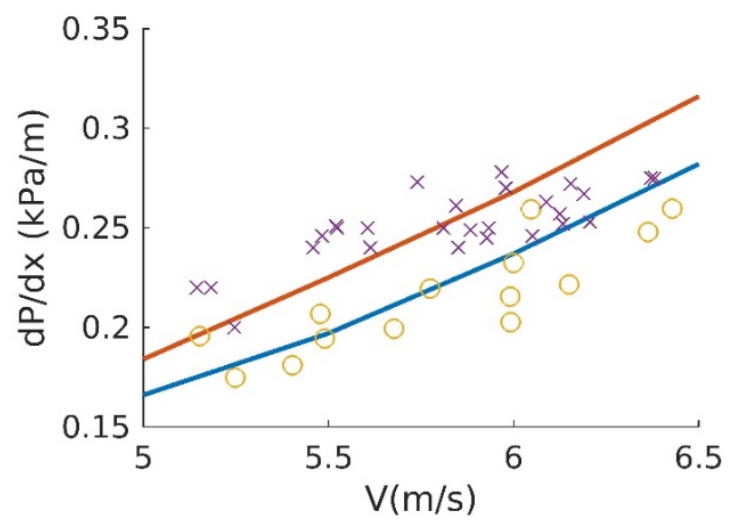

Fig. 13. Pressure gradient for Silin et al(1958) $D=0.900 \mathrm{~m}, \mathrm{~d}=$ $0.250 \mathrm{~mm}, \mathrm{x}: \alpha=20 \%, \circ: \alpha=11 \%$, - : Simulations.

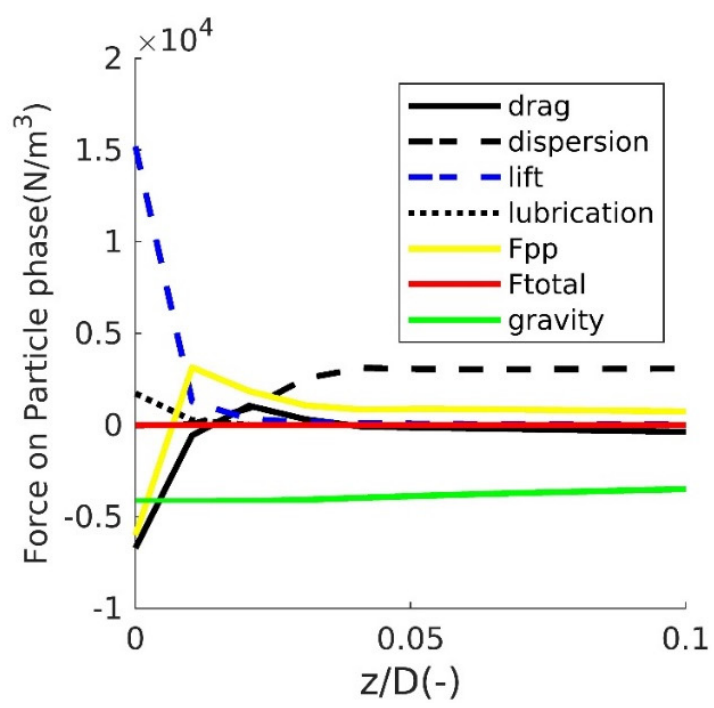

Fig. 14. Forces on the particle phase in vertical direction along the vertical central axis. For $\mathrm{D}=0.103 \mathrm{~m}, \mathrm{dp}=0.270 \mathrm{~mm}$ and $\mathrm{V}=5.4 \mathrm{~m} / \mathrm{s}$. Left: full pipe diameter, right: bottom 0.1 pipe diameter.

\section{Forces on the particle phase}

The advantage of modelling the slurry flow with this twophase model is that all the forces on the particle phase are calculated separately. This gives the opportunity to study these forces. This was also done by Messa et al. (2021). However, this was done for the horizontal direction only for a 2D flow between horizontal plates. In Figure 14 the forces in vertical direction on the particle phase are plotted along the vertical axis in the pipeline. It can be seen that the two main forces acting on the particles are the dispersion force in upward direction and the gravitational force in downward direction. The force on the particle phase due to the kinetic theory and pressure gradient in the vertical direction is plotted as Fpp and gives a contribution in the upward direction.

The last notable force in the middle section of the pipe is the drag force. Contrary to what was expected this gives a contribution in the downward direction. A reason for this can be found in the secondary flow in the plane of the pipe which is shown in Figure 15. The particles move upwards in the middle section of 


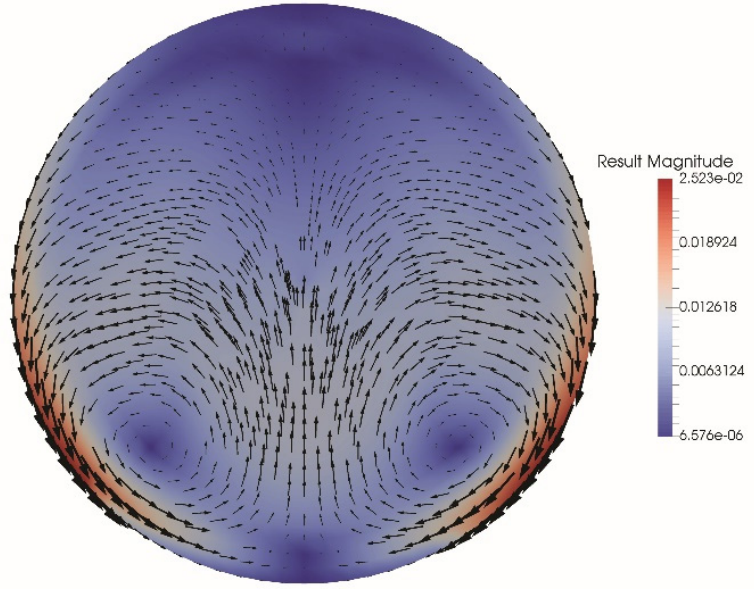

Fig. 15. In plane secundary partcile flow velocity for $D=0.103$ $\mathrm{m}, \mathrm{dp}=0.270 \mathrm{~mm}$ and $\mathrm{V}=5.4 \mathrm{~m} / \mathrm{s}$.

the pipeline. The velocity of this upward movement is bigger than the upwards velocity of the fluid, hence the drag force on the particle phase is pointed downwards. This effect has also been seen in DNS studies by Zheng et al. (2020) and EulerLagrangian simulations by Uzi and Levy (2018). Although the secondary flow velocities seem small in comparison to the mean streamwise flow velocity they amount a lot compared other inplane velocities such as the (hindered) settling velocities.

In the near wall region of the simulation it can be seen that the other forces such as the lift force and the lubrication force have a significant contribution to the vertical particle force but their magnitude decreases quickly when moving away from the wall. Near the bottom wall the contribution of the kinetic theory changes sign and is directed downward resulting in a compaction of the particle fraction due to particle-particle interaction. A similar behaviour has been identified by Capecelatro and Desjardins (2013).

The ratio between the drag and the dispersion force along the vertical axis is given in Figure 16 for three simulated cases. It can be seen that the ratio in the middle section is on average as big as $20 \%$ and therefore the drag force induced by secondary flow in vertical transport is not negligible.

\section{CONCLUSION}

This paper evaluates a two-phase Euler-Euler model that solves for the complete granular temperature equation including transport terms and uses a buoyant k-epsilon model for the turbulence in the fluid phase. The model is solved with second order schemes and the solutions are mesh independent to acceptable level. The model was compared with experimentally determined hydraulic gradients, concentration distributions and velocity distributions in a wide range of operating conditions with variable pipe diameters, particle sizes, concentrations and flow velocities. The influence of various inter phase forces is studied.

The models show satisfactory agreement with concentration profiles in a wide range of flow velocities, particle diameters and pipe diameters. A key parameter for dredging practice is the pressure gradient as a function of the flow velocity, particle size, pipe diameter and in-situ concentration. The pressure gradient was calibrated for by adjusting the specularity coefficient in one calibration case. Hereafter, the same coefficient was used throughout all simulated cases. The maximum error between simulations and experiments is less than $10 \%$ for ex-

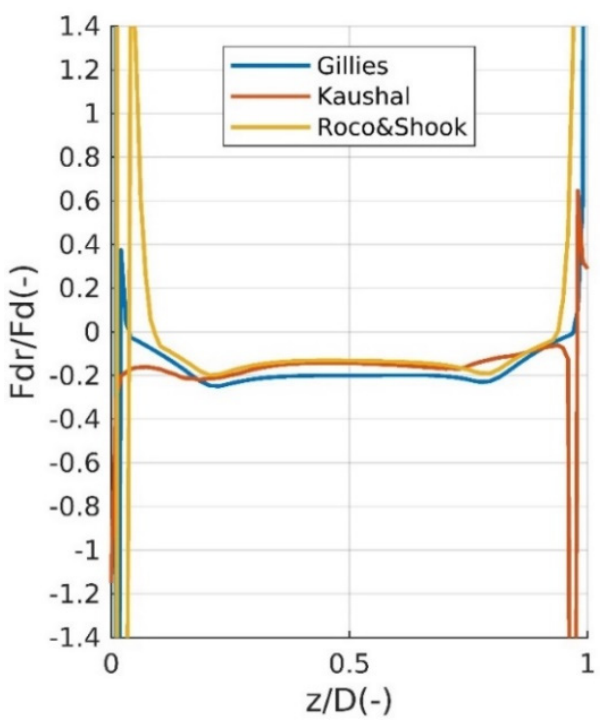

Fig. 16. Ratio of drag force and dispersion force along the vertical central axis. Gillies: for $\mathrm{D}=0.103 \mathrm{~m}, \mathrm{dp}=0.270 \mathrm{~mm}, \alpha=0.3$ and $\mathrm{V}=5.4 \mathrm{~m} / \mathrm{s}$. Kaushal: for $\mathrm{D}=0.0549 \mathrm{~m}, \mathrm{dp}=0.440 \mathrm{~mm}, \alpha=0.33$ and $\mathrm{V}=3 \mathrm{~m} / \mathrm{s}$. Roco and Shook: for $\mathrm{D}=0.263 \mathrm{~m}, \mathrm{dp}=0.165 \mathrm{~mm}$, $\alpha=0.27$ and $\mathrm{V}=3.5 \mathrm{~m} / \mathrm{s}$.

periments considered here. The capability of the model to predict the concentration profile, velocity profile and pressure gradient with satisfactory agreement shows that the model is capable of simulating various suspended slurries.

The dominant upward driving forces that compensate gravitation in the bottom wall region of the pipe are the lift force, collisional force and averaged drag force.

Moving further towards the middle of the pipe the turbulent dispersion forces become the dominant upward driving force as expected. However, the role of the averaged drag force that results from secondary flows is not negligible. This demonstrates that this type of modelling is favourable compared to cross-averaged models that don't take into account such patterns in the cross sections.

For a larger pipe diameter of $0.9 \mathrm{~m}$ the model is validated using pressure gradient measurements. This indicates that the model is not sensitive for scale variation. More detailed experiments on larger pipe diameters is required to validate the performance of the model for industrial practice.

Although Euler-Euler-KT models have a firm theoretical basis calibration of model coefficients is unavoidable. Interestingly, it appears that it was sufficient to select an appropriate value of the specularity coefficient based on a preliminary study to the pressure drop. All the above leads to the conclusion that the present model has the potential to predict suspended slurry flows. And provides a good basis for further research including the simulation other flow regimes such as sliding and fixed beds.

Acknowledgements. This work is part of the research programme Topsector Water with Number ALWTW.2016.050, which is (partly) financed by the Dutch Research Council (NWO).

\section{REFERENCES}

Antal, S.P., Lahey Jr, R.T., Flaherty, J.E., 1991. Analysis of phase distribution in fully developed laminar bubbly twophase flow. International Journal of Multiphase Flow, 17, 5, 635-652. 
Auton, T.R., 1987. The lift force on a spherical body in a rotational flow. Journal of Fluid Mechanics, 183, 199-218.

Bagnold, R.A., 1954. Experiments on a gravity-free dispersion of large solid spheres in a Newtonian fluid under shear. Proceedings of the Royal Society of London. Series A. Mathematical and Physical Sciences, 225, 1160, 49-63.

Behzadi, A., Issa, R.I., Rusche, H., 2004. Modelling of dispersed bubble and droplet flow at high phase fractions. Chemical Engineering Science, 59, 4, 759-770.

Boyer, F., Guazzelli, É., Pouliquen, O., 2011. Unifying suspension and granular rheology. Physical Review Letters, 107, $18,188301$.

Burns, A.D., Frank, T., Hamill, I., Shi, J.M., 2004. The Favre averaged drag model for turbulent dispersion in Eulerian multi-phase flows. In: Proc. 5th international conference on multiphase flow, ICMF (Vol. 4, pp. 1-17). ICMF.

Capecelatro, J., Desjardins, O., 2013. Eulerian-Lagrangian modeling of turbulent liquid-solid slurries in horizontal pipes. International Journal of Multiphase Flow, 55, 64-79.

Chen, L., Duan, Y., Pu, W., Zhao, C., 2009. CFD simulation of coal-water slurry flowing in horizontal pipelines. Korean Journal of Chemical Engineering, 26, 4, 1144-1154.

DallaValle, J.M., 1948. Micromeritics, the Technology of Fine Particles. 2nd Ed. Pitman Pub. Corp., New York.

Di Felice, R., 1994. The voidage function for fluid-particle interaction systems. International Journal of Multiphase Flow, 20, 1, 153-159.

Durand, R., 1953. Basic relationships of the transportation of solids in pipes-experimental research. In: Proceedings of the $5^{\text {th }}$ Congress of International Association of Hydraulic Research. Minneapolis.

Ekambara, K., Sanders, R.S., Nandakumar, K., Masliyah, J.H., 2009. Hydrodynamic simulation of horizontal slurry pipeline flow using ANSYS-CFX. Industrial \& Engineering Chemistry Research, 48, 17, 8159-8171.

Gidaspow, D., 1994. Multiphase Flow and Fluidization: Continuum and Kinetic Theory Descriptions. Academic Press.

Gillies, R.G., Shook, C.A., 1994. Concentration distributions of sand slurries in horizontal pipe flow. Particulate Science and Technology, 12, 1, 45-69.

Gillies, R.G., Shook, C.A., 2000. Modelling high concentration settling slurry flows. The Canadian Journal of Chemical Engineering, 78, 4, 709-716.

Gillies, R.G., Shook, C.A., Xu, J., 2004. Modelling heterogeneous slurry flows at high velocities. The Canadian Journal of Chemical Engineering, 82, 5, 1060-1065.

Goeree, J.C., Keetels, G.H., Munts, E.A., Bugdayci, H.H., van Rhee, C., 2016. Concentration and velocity profiles of sediment $\square$ water mixtures using the drift flux model. The Canadian Journal of Chemical Engineering, 94, 6, 1048-1058.

Gopaliya, M.K., Kaushal, D.R., 2015. Analysis of effect of grain size on various parameters of slurry flow through pipeline using CFD. Particulate Science and Technology, 33, 4, 369-384.

Gopaliya, M.K., Kaushal, D.R., 2016. Modeling of sand-water slurry flow through horizontal pipe using CFD. Journal of Hydrology and Hydromechanics, 64, 3, 261-272.

Gosman, A.D., Lekakou, C., Politis, S., Issa, R.I., Looney, M.K., 1992. Multidimensional modeling of turbulent two phase flows in stirred vessels. AIChE Journal, 38, 12, 19461956.

Greenshields, C.J., 2015. OpenFOAM user guide. OpenFOAM Foundation Ltd, Version 3(1), 47 p

Henkes, R.A.W.M., Van Der Vlugt, F.F., Hoogendoorn, C.J., 1991. Natural-convection flow in a square cavity calculated with low-Reynolds-number turbulence models. International Journal of Heat and Mass Transfer, 34, 2, 377-388.

Ismail, H.M., 1952. Turbulent transfer mechanism of suspended sediment in closed channels. Trans. ASCE, 117, 1.

Jenkins, J.T., Savage, S.B., 1983. Theory for the rapid flow of identical, smooth, nearly elastic, spherical particles. Journal of Fluid Mechanics, 130, 1, 187-202.

Johnson, P.C., Jackson, R., 1987. Frictional-collisional constitutive relations for granular materials, with application to plane shearing. Journal of Fluid Mechanics, 176, 67-93.

Kaushal, D.R., Tomita, Y., 2003. Comparative study of pressure drop in multisized particulate slurry flow through pipe and rectangular duct. International Journal of Multiphase Flow, 29, 9, 1473-1487.

Kaushal, D.R., Tomita, Y., 2007. Experimental investigation for near-wall lift of coarser particles in slurry pipeline using $\gamma$-ray densitometer. Powder Technology, 172, 3, 177-187.

Kaushal, D.R., Thinglas, T., Tomita, Y., Kuchii, S., Tsukamoto, H., 2012. CFD modeling for pipeline flow of fine particles at high concentration. International Journal of Multiphase Flow, 43, 85-100.

Koch, D.L., 1990. Kinetic theory for a monodisperse gas-solid suspension. Physics of Fluids A: Fluid Dynamics, 2, 10, $1711-1723$

Kumar, N., Gopaliya, M.K., Kaushal, D.R., 2016. Modeling for slurry pipeline flow having coarse particles. Multiphase Science and Technology, 28, 1.

Kumar, N., Gopaliya, M.K., Kaushal, D.R., 2019. Experimental investigations and CFD modeling for flow of highly concentrated iron ore slurry through horizontal pipeline. Particulate Science and Technology, 37, 2, 232-250.

Launder, B.E., Spalding, D.B., 1974. The numerical computation of turbulent flows. Computer Methods in Applied Mechanics and Engineering, 3, 2, 269-289.

Legendre, D., Magnaudet, J., 1997. A note on the lift force on a spherical bubble or drop in a low-Reynolds-number shear flow. Physics of Fluids, 9, 11, 3572-3574.

Legendre, D., Magnaudet, J., 1998. The lift force on a spherical bubble in a viscous linear shear flow. Journal of Fluid Mechanics, 368, 81-126.

Louge, M.Y., Mastorakos, E., Jenkins, J.T., 1991. The role of particle collisions in pneumatic transport. Journal of Fluid Mechanics, 231, 8, 345.

Lun, C.K.K., 1991. Kinetic theory for granular flow of dense, slightly inelastic, slightly rough spheres. Journal of Fluid Mechanics, 233, 539-559.

Matousek, V., 2002. Pressure drops and flow patterns in sandmixture pipes. Experimental Thermal and Fluid Science, 26, 6-7, 693-702.

Matoušek, V., 2009. Predictive model for frictional pressure drop in settling-slurry pipe with stationary deposit. Powder Technology, 192, 3, 367-374.

McLaughlin, J.B., 1991. Inertial migration of a small sphere in linear shear flows. J. Fluid Mech., 224, 261-274, 332.

Messa, G.V., Matoušek, V., 2020. Analysis and discussion of two fluid modelling of pipe flow of fully suspended slurry. Powder Technology, 360, 747-768.

Messa, G.V., Malin, M., Malavasi, S., 2014. Numerical prediction of fully-suspended slurry flow in horizontal pipes. Powder Technology, 256, 61-70.

Messa, G.V., Malin, M., Matoušek, V., 2021. Parametric study of the $\beta-\sigma$ two-fluid model for simulating fully suspended slurry flow: effect of flow conditions. Meccanica, 5, 1-31.

O'Brien, M.P., 1933. Review of the theory of turbulent flow and its relation to sediment transportation. Eos, Transactions 
American Geophysical Union, 14, 1, 487-491.

Roco, M.C., Shook, C.A., 1983. Modeling of slurry flow: the effect of particle size. The Canadian Journal of Chemical Engineering, 61, 4, 494-503.

Rodi, W., 1993. Turbulence Models and Their Application in Hydraulics. $2^{\text {nd }} E d$. CRC Press.

Rouse, H., 1937. Modern conceptions of the mechanics of fluid turbulence. Trans ASCE, 102, 463-505.

Segre, G., Silberberg, A., 1962. Behavior of macroscopic rigid spheres in Poiseuille flow. J. Fluid Mech, 14.

Schaan, J., Sumner, R.J., Gillies, R.G., Shook, C.A., 2000. The effect of particle shape on pipeline friction for Newtonian slurries of fine particles. The Canadian Journal of Chemical Engineering, 78, 4, 717-725.

Shook, C.A., Daniel, S.M., 1965. Flow of suspensions of solids in pipelines: Part I. Flow with a stable stationary deposit. The Canadian Journal of Chemical Engineering, 43, 2, 5661.

Shook, C.A., Daniel, S.M., Scott, J.A., Holgate, J.P., 1968. Flow of suspensions in pipelines (Part 2: Two mechanisms of particle suspension). The Canadian Journal of Chemical Engineering, 46, 4, 238-244.

Silin, M.O., Kobernik, S.G., Asaulenko, I.A., 1958. Druckhohenverluste von Wasser und Wasser-Boden-Gemischen in Rohrleitungen grossen Durchmessers. Dopovidi Natsional'noi Akademii nauk Ukrainy, 2, 175-177.
Syamlal, M., Rogers, W., OBrien, T.J., 1993. MFIX documentation theory guide (No. DOE/METC-94/1004). USDOE Morgantown Energy Technology Center, WV (United States).

Ting, X., Miedema, S.A., Xiuhan, C., 2019. Comparative analysis between CFD model and DHLLDV model in fullysuspended slurry flow. Ocean Engineering, 181, 29-42.

Turian, R.M., Yuan, T.F., 1977. Flow of slurries in pipelines. AIChE Journal, 23, 3, 232-243.

Uzi, A., Levy, A., 2018. Flow characteristics of coarse particles in horizontal hydraulic conveying. Powder Technology, 326, 302-321.

van Wachem, B.G.M., 2000. CFD simulations of gas-solid fluidised beds. PhD Thesis in Chemical Engineering. Delf University of Technology, Delft.

Wilson, K.C., Clift, R., Sellgren, A., 2002. Operating points for pipelines carrying concentrated heterogeneous slurries. Powder Technology, 123, 1, 19-24.

Zhang, D.Z., Rauenzahn, R.M., 1997. A viscoelastic model for dense granular flows. Journal of Rheology, 41, 6, 1275-1298.

Zheng, E., Rudman, M., Kuang, S., Chryss, A., 2020. Turbulent coarse-particle suspension flow: Measurement and modelling. Powder Technology, 373, 647-659.

Received 14 January 2021 Accepted 27 April 2021 